\title{
Haloperidol Prophylaxis for Elderly Hip-Surgery Patients at Risk for Delirium: A Randomized Placebo-Controlled Study
}

\author{
Kees J. Kalisvaart, MD, ${ }^{*}$ Jos F. M. de Jonghe, PhD, ${ }^{*}$ Marja J. Bogaards, PharmD, ${ }^{\dagger}$ \\ Ralph Vreeswijk, RN, MSc, ${ }^{*}$ Toine C. G. Egberts, PhD, ${ }^{\ddagger}$ Bart J. Burger, MD, PhD, *

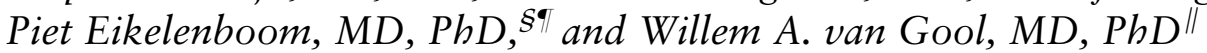

\begin{abstract}
OBJECTIVES: To study the effectiveness of haloperidol prophylaxis on incidence, severity, and duration of postoperative delirium in elderly hip-surgery patients at risk for delirium.
\end{abstract}

DESIGN: Randomized, double-blind, placebo-controlled trial. SETTING: Large medical school-affiliated general hospital in Alkmaar, the Netherlands.

PARTICIPANTS: A total of 430 hip-surgery patients aged 70 and older at risk for postoperative delirium.

INTERVENTION: Haloperidol $1.5 \mathrm{mg} / \mathrm{d}$ or placebo was started preoperatively and continued for up to 3 days postoperatively. Proactive geriatric consultation was provided for all randomized patients.

MEASUREMENTS: The primary outcome was the incidence of postoperative delirium (Diagnostic and Statistical Manual of Mental Disorders, Fourth Edition, and Confusion Assessment Method criteria). Secondary outcomes were the severity of delirium (Delirium Rating Scale, revised version-98 (DRS-R-98)), the duration of delirium, and the length of hospital stay.

RESULTS: The overall incidence of postoperative delirium was $15.8 \%$. The percentage of patients with postoperative delirium in the haloperidol and placebo treatment condition was $15.1 \%$ and $16.5 \%$, respectively (relative risk $=0.91,95 \%$ confidence interval $(\mathrm{CI})=0.6-1.3)$; the mean highest DRS-R-98 score \pm standard deviation was

From the *Departments of Geriatric Medicine and Orthopedic Surgery, Medical Center Alkmaar, Alkmaar, the Netherlands; ${ }^{\dagger}$ Department of Pharmacy, Reinier de Graaf Group, Delft, the Netherlands; ${ }^{\ddagger}$ Department of Pharmacoepidemiology and Pharmacotherapy, Utrecht Institute for Pharmaceutical Sciences, Utrecht University, the Netherlands; Departments of SPsychiatry and "Neurology, Academic Medical Center, University of Amsterdam, Amsterdam, the Netherlands; and "Free University of Amsterdam, Amsterdam, the Netherlands.

A paper with the preliminary results was accepted for presentation at the meeting of the American Geriatrics Society in Baltimore, Maryland, 2003. Because of illness, no presentation was made. Abstract was published in the Journal of the American Geriatrics Society, May 2003.

Address correspondence to K. J. Kalisvaart, MD, Medical Center Alkmaar, PO Box 501, 1800 AM Alkmaar, the Netherlands.

E-mail: k.kalisvaart@mca.nl

DOI: $10.1111 / \mathrm{j} .1532-5415.2005 .53503 . \mathrm{x}$
$14.4 \pm 3.4$ and $18.4 \pm 4.3$, respectively (mean difference $4.0,95 \% \mathrm{CI}=2.0-5.8 ; P<.001)$; delirium duration was 5.4 versus 11.8 days, respectively (mean difference 6.4 days, $95 \% \mathrm{CI}=4.0-8.0 ; P<.001)$; and the mean number of days in the hospital was $17.1 \pm 11.1$ and $22.6 \pm 16.7$, respectively (mean difference 5.5 days, 95\% $\mathrm{CI}=1.4-2.3$; $P<.001)$. No haloperidol-related side effects were noted.

CONCLUSION: Low-dose haloperidol prophylactic treatment demonstrated no efficacy in reducing the incidence of postoperative delirium. It did have a positive effect on the severity and duration of delirium. Moreover, haloperidol reduced the number of days patients stayed in the hospital, and the therapy was well tolerated. J Am Geriatr Soc 53:1658-1666, 2005.

Key words: haloperidol; delirium prevention; prophylaxis; elderly; orthopedic surgery; risk factors; delirium severity; duration of hospital stay; assessment of delirium

$\mathrm{D}$ elirium is a serious postoperative complication in elderly patients. ${ }^{1-3}$ It is associated with high morbidity and mortality, increased length of hospital stay, and a high rate of institutionalization after discharge..$^{2,4-7}$ Incidence rates for delirium of $5 \%$ to $45 \%$ in patients undergoing orthopedic hip surgery emphasize the need for primary and secondary prevention. ${ }^{2,8,9}$

Although delirium can occur in any older patient, some are more at risk than others. Many predisposing and precipitating factors have been identified (e.g., cognitive impairment, sensory impairment, severity of illness, and dehydration). ${ }^{10}$ To counteract the effect of some of these risk factors, previous studies on delirium prevention have focused on nonpharmacological interventions such as reorienting the patient, modifying the hospital environment, proactive geriatric consultation, pain treatment programs, family education, early mobilization protocol, nutritional support, and infection control measures. ${ }^{11-15} \mathrm{~A}$ meta-analysis revealed that, on average, nonpharmacological interventions reduce the absolute risk of delirium by a mere $13 \% .{ }^{16}$ Further reduction of the incidence, severity, and duration of postoperative delirium could have an 
important effect on the burden of surgical procedures in elderly patients, but little is known about the effectiveness of prophylactic pharmacological treatment strategies for postoperative delirium.

The antipsychotic drug haloperidol is widely used for the symptomatic treatment of delirium. ${ }^{17}$ In one small study, haloperidol prophylaxis proved to be effective in reducing delirium in gastrointestinal surgery patients. ${ }^{18}$

Haloperidol is a dopamine antagonist. Dopamine D2 receptor blockade is associated with enhanced acetylcholine release. ${ }^{19,20}$ Delirium is highly associated with cholinergic deficiency. So it can be hypothesized that haloperidol may have an indirect beneficial effect on delirium. Indeed, some dopamine receptor antagonists, particularly antipsychotics, appear to treat delirium, including that arising from anticholinergic causes. ${ }^{21,22}$

In other conditions that are associated with cholinergic deficiency, such as Alzheimer's disease, haloperidol and physostigmine have a positive effect on delusions and hallucinations, which are symptoms of delirium as well. ${ }^{23}$ The documented therapeutic effects, as well as its pharmacological profile and a possible "priming" effect, suggest that haloperidol could prevent the occurrence of delirium or reduce its severity or duration, but no controlled studies have evaluated the prophylactic effect of haloperidol on postoperative delirium. There are potential side effects: hypotension (minimal), particularly with parenteral administration; sedation; altering of cardiac conduction; and extrapyramidal symptoms. In addition, haloperidol has a lower potency of cholinergic blockade than other neuroleptics. Keeping the total daily dose of haloperidol below $3 \mathrm{mg}$ may reduce the risk of extrapyramidal side effects. ${ }^{24}$

This was a randomized, placebo-controlled, doubleblind, clinical trial of low-dose haloperidol prophylaxis for postoperative delirium in elderly hip-surgery patients who were at intermediate or high risk for this complication. The aim was to assess the effectiveness of $1.5 \mathrm{mg}$ of haloperidol daily versus placebo on the primary (incident delirium) and secondary (deterioration of delirium) prevention of postoperative delirium in hip-surgery patients.

\section{METHODS}

\section{Ethical Considerations}

The study was undertaken in accordance with the Declaration of Helsinki and the guidelines on good clinical practice. Approval of the regional research ethics committee was obtained. All patients or their relatives gave fully informed written consent.

\section{Study Design and Objectives}

This was a randomized, placebo-controlled, double-blind, clinical trial, with a minimum duration of 1 day and a maximum of 6 days, depending on the onset of delirium. The study aim was to evaluate efficacy of $1.5 \mathrm{mg}$ of haloperidol daily versus placebo on the primary and secondary prevention of postoperative delirium in elderly hip-surgery patients.

\section{Participants}

A research team of geriatricians and nurses in a single 915bed teaching hospital in the Netherlands identified potentially eligible patients by systematically screening new admissions to two surgical and three orthopedic wards. Men and women aged 70 and older admitted for acute or elective hip surgery were considered for inclusion in the haloperidol prophylaxis study, provided that they were at intermediate or high risk for postoperative delirium. Risk classification was based on the presence of four predictive risk factors, as described elsewhere. ${ }^{10,25}$ Visual impairment, defined as binocular near vision worse than 20/70 after correction; severity of illness, measured using the Acute Physiology Age and Chronic Health Examination (APACHE II) scale of 0 to $70,{ }^{26}$ with a cut-off score of 16 or higher indicating increased severity; cognitive impairment (Mini-Mental State Examination (MMSE) score $\leq 24$ on a scale of $0-30) ;{ }^{27}$ and index of dehydration (ratio of blood urea nitrogen to creatinine of $\geq 18$ ). Intermediate risk for postoperative delirium was defined as presence of one or two risk factors and high risk as presence of three or more risk factors. The low-risk patients were assessed daily according to the protocol for incident delirium but received no prophylactic medication.

Patients were ineligible if they had delirium at admission, no risk factors for postoperative delirium present at baseline, history of haloperidol allergy, use of cholinesterase inhibitors, parkinsonism, epilepsy, levodopa treatment, inability to participate in interviews (profound dementia, language barrier, intubation, respiratory isolation, aphasia, coma, or terminal illness), delay of surgery of more than 72 hours after admission, or a prolonged QTc interval of $460 \mathrm{~ms}$ or higher for men and $470 \mathrm{~ms}$ or higher for women on their electrocardiogram.

Eligibility was checked against patients' clinical notes and their own recall. Patients were randomized between August 2000 and August 2002.

\section{Measurements and Procedures}

Eligible patients were sequentially randomly assigned to study treatment (placebo or haloperidol $0.5 \mathrm{mg}$ three times daily) from a block of drugs that the hospital pharmacist had prepackaged, according to a computer-generated randomization code. Placebo medication was identical in appearance to the active drug. The research team and all participants were blinded to the treatment group, and blinding was maintained throughout the study and checked by interviewing the assessors.

Trial medication was started on admission and continued until 3 days after surgery. A maximum delay for surgery of 72 hours was permitted. The haloperidol dosage was based on the average starting dose for treatment of older patients with delirium in the department and recommendations by the American Psychiatric Association. ${ }^{17}$ All patients were assessed daily for efficacy and safety evaluations. Experienced geriatric nurses and geriatricians provided proactive geriatric consultation to all patients. The consultation was based on a structured multimodular protocol (geriatric medical attention; enhancement of orientation and cognition; sensory and mobility-improving advice; attention to pain and sleeping 
problems; extra attention to fluid and food intake; and patient, family, and nursing staff education). If postoperative delirium occurred, patients were treated according to standard procedures (haloperidol three times per day, lorazepam three times per day, or both in increasing doses, depending on symptoms of delirium) and assessed for delirium severity and duration.

Code envelopes were stored in the pharmacy and at the investigation site. In case of emergency, an independent physician could request unmasking of the treatment allocation. A statement had to be made in the Case Report Formulary after breaking the seal. This happened with two patients in the haloperidol group and five in the placebo group. These patients do not appear in the protocol violation count (Figure 1). In all other cases, the treatment was blinded until the end of the total study.

The clinical staff - independent of the research staffrecorded the level of adherence to the intervention, with reasons for nonadherence, daily. Adherence was complete if the patient received all medication at the times it had to be given. Partial adherence indicated that the patient received some but not all the medication or not at the scheduled times. Nonadherence indicated that none of the medication was received that day.

\section{Assessment}

Members of the research team not involved in the clinical care of the patients performed all baseline and outcome assessments. Assessors had extensive training before the study and followed standard procedures. All data were collected on standardized patient record forms and underwent extensive checks of error and validity.

The baseline screening and assessments were completed before surgery and within 12 hours after admission and included the MMSE, the Informant Questionnaire on Cognitive Decline in the Elderly (measures preexisting cognitive impairment), ${ }^{28}$ the standardized Snellen test for visual impairment ${ }^{29}$ chart review to determine APACHE II score (range $0=$ no acute health problems to $70=$ severe acute health problems), ratio of blood urea nitrogen to creatinine, Geriatric Depression Scale (15-item version selfrating scale for depression, range $0-15$, higher scores indicating depression), ${ }^{30}$ and the Barthel Index (range 0-20, lower scores indicating more dependency). ${ }^{31}$ Safety was monitored throughout the study and was based on analysis of adverse events, daily examination by the treating surgeons, spontaneous reports from the patients, and specific assessments; the Barnes Akathisia Scale (range 0 (no akathisia) to 14 (severe akathisia) was used to assess drug-induced akathisia. ${ }^{32}$ Electrocardiogram was performed on admission and in case of an adverse event, for evaluation of QTc interval. Daily blood pressure measurements were taken to check for postural hypotension. Patients were clinically assessed daily for signs of sedation and extrapyramidal signs.

\section{Outcomes}

The primary outcome was postoperative delirium. Diagnosis of the syndrome was defined using Diagnostic and Statistical Manual of Mental Disorders, Fourth Edition (DSM-IV) and Confusion Assessment Method (CAM) criteria. ${ }^{33,34}$ Second- ary outcome variables were severity of delirium, delirium duration, and length of hospital stay. Delirium severity was measured using the Delirium Rating Scale, revised version98 (DRS-R-98, range 0 (no severity) to 45 (high severity)). ${ }^{35}$ Daily patients assessments using the MMSE, DRS-R-98, and Digit Span test (assessment of attention, range 0 (no attention) to 42 (good attention) ${ }^{36}$ were used to make the DSM-IV and CAM diagnoses possible and to assess delirium severity. CAM and DRS-R-98 assessments were continued once delirium was diagnosed.

\section{Statistical Analysis}

Statistical calculations were performed using SPSS for Windows, version 11 (SPSS, Inc., Chicago, IL). Calculation of the required sample size was based on the assumption that haloperidol prophylaxis would reduce the incidence of postoperative delirium from $40 \%$ to $27 \%$. These figures were based on a $40 \%$ incidence of delirium in a comparable population in the pretrial period in the study hospital in 1999 and an absolute $13 \%$ median risk reduction as found in studies using nonpharmacological interventions. ${ }^{16}$ With a two-sided test, an alpha level of 0.05 , and a power of $80 \%$, the analysis required 206 patients per group. The analysis was undertaken as intention to treat at all levels. The primary outcome of the study was the incidence of postoperative delirium, defined according to the DSM-IV criteria.

For the primary analysis of the intervention, delirium was considered a binary outcome (absent or present) according to its earliest occurrence. Secondary outcomes were the severity of delirium, which was measured using the DRS-R-98 and was expressed as the maximum DRS-R-98 score during the delirium period, the duration of delirium, and duration of hospital stay of delirious patients (the number of days spent in hospital until patients were ready for transfer to a rehabilitation unit or home).

Proportions of patients were compared using the Fisher exact test. Two-tailed $P$-values $<.05$ were considered to indicate statistical significance. Parametric and nonparametric values were tested using Student $t$ test and the MannWhitney $U$ test, respectively. The results are expressed as relative risks with $95 \%$ confidence intervals (CIs) for the haloperidol group relative to the placebo group, with a relative risk less than 1.0 indicating a beneficial effect.

\section{RESULTS}

Of 681 individuals initially admitted to the orthopedic and surgical units, 603 entered the baseline phase (Figure 1). Failure to meet the inclusion criteria was the most typical reason for not entering this phase $(\mathrm{n}=78) ; 36$ refused to participate, 13 were discharged without surgery, eight could not be tested, six had surgery before testing could take place, six were known to have parkinsonism, four were taking antipsychotic drugs, three were missed by failure of the emergency department staff to report them, one had extreme liver failure, and one was delirious at admission. One hundred twenty-one of 603 patients were not randomized because all four risk factors were absent (low risk). Of the 482 entering the baseline phase, 52 refused to comply after baseline screening, all because they or their caregiver refused treatment with the study drug. The remaining 430 eligible patients were randomized (Figure 1). 


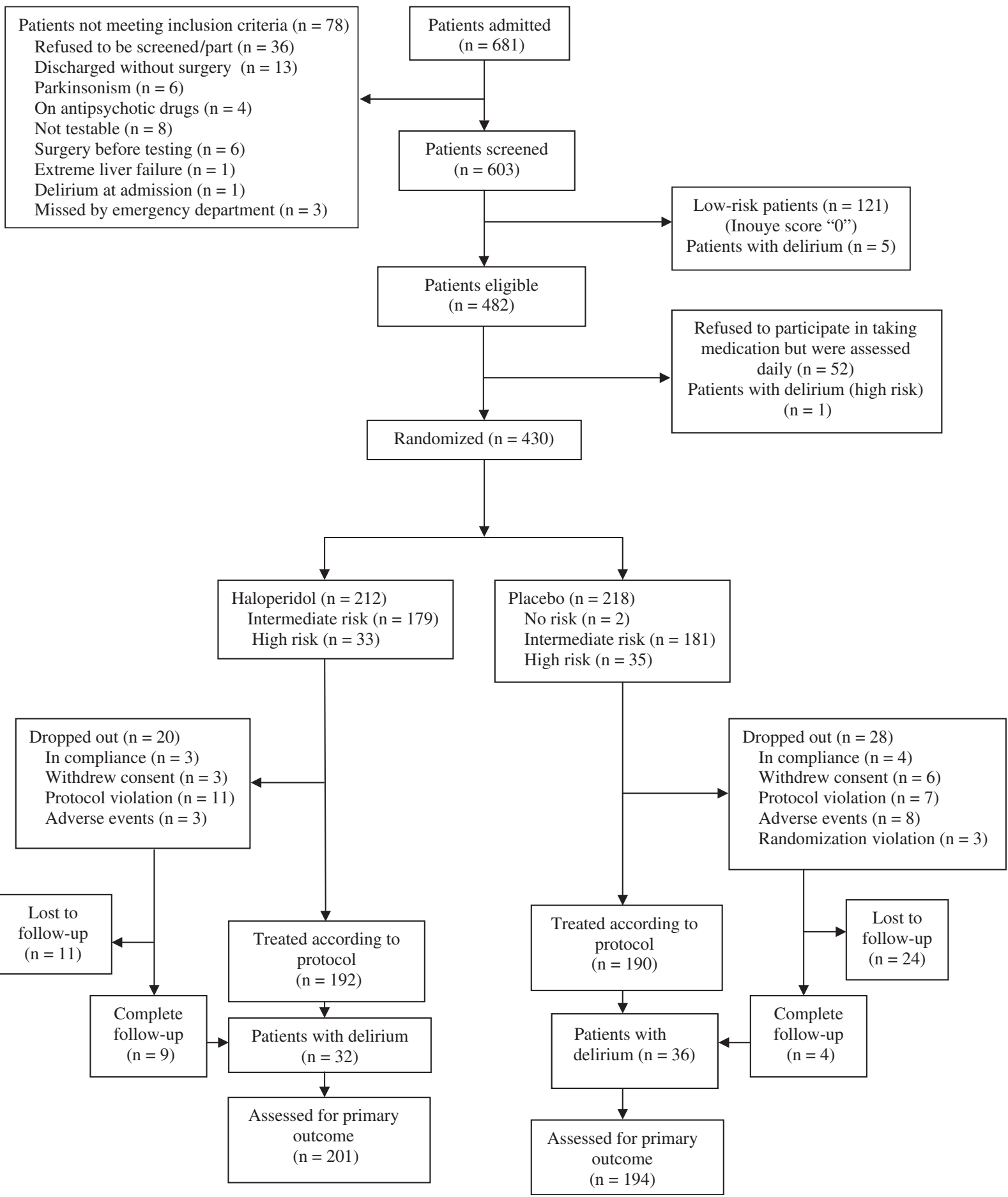

Figure 1. Flow diagram of the study.

\section{Subject Profile}

The characteristics at the time of admission of the 212 patients randomized to haloperidol prophylaxis and the 218 patients in the placebo group are shown in Table 1 . The groups did not differ significantly in terms of any of these characteristics. The mean number of risk factors per patient was similar in the two groups. On average, both study groups included elderly patients with minimal cognitive impairment, some visual impairment, and light dehydration. MMSE results at baseline did not differ between elective and fracture patients. Patients with an intermediate baseline risk for delirium and nonacute (elective) surgery were overrepresented, indicating that, overall, the study group was in relatively good clinical condition. The low APACHE II scores and the high Barthel Index scores are consistent with this.

\section{Primary Outcome: Incidence of Delirium}

Intention-to-treat (ITT) analysis included 430 patients; delirium occurred in $68(15.8 \%)$. The incidence of delirium 
Table 1. Characteristics of the Patients on Admission According to Study Group/Intention-to-Treat Group

\begin{tabular}{|c|c|c|c|}
\hline Characteristic & $\begin{array}{l}\text { Haloperidol } \\
(\mathrm{n}=212)\end{array}$ & $\begin{array}{l}\text { Placebo } \\
(n=218)\end{array}$ & $P$-value \\
\hline Age, mean $\pm S D$ & $78.71 \pm 6.04$ & $79.57 \pm 6.27$ & .15 \\
\hline Female, n (\%) & $172(81.1)$ & $171(78.9)$ & .56 \\
\hline Mini-Mental State Examination score, mean $\pm \mathrm{SD}^{*}$ & $24.97 \pm 6.85$ & $24.52 \pm 4.20$ & .96 \\
\hline Visual acuity, mean $\pm \mathrm{SD}^{\dagger}$ & $0.44 \pm 0.16$ & $0.40 \pm 0.16$ & .79 \\
\hline Acute Physiology Age and Chronic Health Examination II score, mean $\pm \mathrm{SD}^{\ddagger}$ & $13.44 \pm 3.2$ & $13.27 \pm 3.1$ & .57 \\
\hline Blood urea nitrogen/creatinine ratio, mean $\pm \mathrm{SD}^{\S}$ & $20.8 \pm 4.0$ & $20.45 \pm 3.5$ & .47 \\
\hline Geriatric Depression Scale-15 score, mean $\pm \mathrm{SD}^{\|}$ & $1.09 \pm 1.4$ & $1.29 \pm 1.7$ & .17 \\
\hline Barthel Index, mean $\pm \mathrm{SD}^{\uparrow}$ & $18.60 \pm 1.3$ & $18.96 \pm 2.5$ & .14 \\
\hline \multicolumn{4}{|l|}{ Baseline risk of delirium, $\mathrm{n}(\%)$} \\
\hline Intermediate & $179(84.4)$ & $181(83.3)^{\#}$ & .64 \\
\hline High & $33(15.6)$ & $35(16.1)$ & .89 \\
\hline \multicolumn{4}{|l|}{ Admission, $\mathrm{n}(\%)$} \\
\hline Acute (fracture) & $53(25.0)$ & $60(27.6)$ & .65 \\
\hline Elective (replacement) & $159(75.0)$ & $157(72.4)$ & .43 \\
\hline Hospital days, mean \pm SD & 13. $8 \pm 7.7$ & $13.6 \pm 7.8$ & .84 \\
\hline
\end{tabular}

Note: Because of rounding, percentages may not total 100.

${ }^{*}$ Range 0 (severe cognitive impairment) to 30 (no cognitive impairment).

${ }^{\dagger}$ Range 20/20 (no visual impairment) to 20/800 (severe visual impairment).

${ }^{\ddagger}$ Range 0 (no acute health problems) to 70 (severe acute health problems).

${ }^{\S}$ Ratio greater than 18 indicates dehydration.

"Range 0 (depression not likely) to 15 (depression very likely).

"Range 0 (severe disability) to 20 (no disability).

\# Two patients with no risk missing from total 218 patients in placebo group; see Figure 1.

$\mathrm{SD}=$ standard deviation

in the ITT haloperidol group of $15.1 \%(32 / 212)$ did not differ significantly from the $16.5 \%(36 / 218)$ in the placebo group (relative risk $=0.91,95 \% \mathrm{CI}=0.59-1.44$ ) (Table 2). The baseline characteristics of patients in the haloperidol and placebo group who developed delirium on follow-up did not differ significantly (Table 2).

Forty-four of the randomized 367 patients with an "intermediate risk" for delirium developed delirium $(12 \%$, $95 \% \mathrm{CI}=8.7-15.3 \%)$, whereas 24 of 63 high-risk patients became delirious $(38 \%, 95 \% \mathrm{CI}=26.1-51.2 \%)$. Only five of the 121 low-risk patients $(4.1 \%, 95 \% \mathrm{CI}=0-4.4 \%)$ developed delirium. Per-protocol analysis included 382 patients; delirium occurred in 55 (14.4\%). The dropout incidence was $20(9.4 \%)$ patients in the haloperidol group, of which 11 were lost for follow-up for the per-protocol analysis and $28(12.8 \%)$ patients in the placebo group, of which 24 were lost to follow-up (Figure 1).

\section{Secondary Outcomes}

The characteristics of the episodes of delirium that occurred were markedly different in both groups. The severity of delirium characterized by the highest value of the DRSR-98 (DRS-Max) during an episode with delirium in patients from the haloperidol group was on average \pm standard deviation $14.40 \pm 3.5$, versus $18.41 \pm 4.4$ in the placebo group (mean difference $4.0,95 \% \mathrm{CI}=2.0$ 5.8; $P<.001$ ) (Table 3). During the first 3 days after the onset of delirium, the severity as measured using the mean of the DRS-R-98 scores was significantly lower in patients who had received haloperidol preoperatively (Figure 2), and from Day 5 until Day 8, the proportion of patients still having delirium was significantly lower after haloperidol prophylaxis (Figure 3). The mean duration of delirium in the haloperidol group was 6.4 days $(95 \% \mathrm{CI}=4.0-8.0$; $P<.001$ ) shorter than in the placebo group (haloperidol $5.41 \pm 4.91$ days vs placebo $11.85 \pm 7.56$ days). The mean difference of days spent in the hospital until patients were ready for transfer to a rehabilitation unit or home was 5.5 days shorter $(95 \% \mathrm{CI}=1.4-2.3 ; P<.001)$ in patients from the haloperidol group $(17.1 \pm 11.1)$ than in the placebo group (22.6 \pm 16.7$)$ (Table 3$)$. No episodes with recurrence of delirium were observed in this study.

No drug-related side effects were seen during the study period. The adverse events were never related to extrapyramidal symptoms. (Values on the Barnes Akathisia Scale were 0 for all the patients in both groups.) There was no sedation reported, other than related to the use of morphinomimetics.

There was partial or nonadherence in two patients in the haloperidol group and patients in the placebo group (Figure 1).

\section{DISCUSSION}

Low-dose haloperidol prophylaxis was not effective for the prevention of postoperative delirium in elderly hip-surgery patients at intermediate or high risk for this complication, but haloperidol prophylaxis markedly reduced severity and duration of postoperative delirium. As a result, the burden of postoperative delirium was less, as was the number of days patients stayed in the hospital. No drug-related side effects were noted. 
Table 2. Characteristics of Patients Who Developed Delirium, According to Study Group: Intention-to-Treat Group

\begin{tabular}{|c|c|c|c|}
\hline Characteristic & $\begin{array}{l}\text { Haloperidol } \\
\quad(n=32)\end{array}$ & $\begin{array}{l}\text { Placebo } \\
(n=36)\end{array}$ & $P$-value \\
\hline Age, mean \pm SD & $82.6 \pm 5.3$ & $82.2 \pm 7.4$ & .78 \\
\hline Female, $\mathrm{n}(\%)$ & $24(75.0)$ & $23(63.9)$ & .32 \\
\hline Mini-Mental State Examination score, mean \pm SD* $^{*}$ & $21.7 \pm 3.9$ & $20.5 \pm 4.7$ & .28 \\
\hline Visual acuity, mean $\pm \mathrm{SD}^{\dagger}$ & $.31 \pm .13$ & $0.37 \pm 0.14$ & .07 \\
\hline Acute Physiology Age and Chronic Health Examination II score, mean $\pm S \mathrm{SD}^{\ddagger}$ & $15.7 \pm 3.85$ & $14.6 \pm 3.5$ & .22 \\
\hline Blood urea nitrogen/creatinine ratio, $\pm \mathrm{SD}^{\S}$ & $20.0 \pm 6.67$ & $21.4 \pm 6.1$ & .37 \\
\hline Geriatric Depression Scale-15 score, mean $\pm S D^{\|}$ & $1.4 \pm 1.4$ & $1.6 \pm 1.4$ & .60 \\
\hline Barthel Index, mean $\pm \mathrm{SD}^{\top}$ & $18.1 \pm 2.6$ & $18.5 \pm 3.5$ & .59 \\
\hline \multicolumn{4}{|l|}{ Baseline risk of delirium, $\mathrm{n}(\%)$} \\
\hline Intermediate & $20(62.5)$ & $23(63.9)$ & .91 \\
\hline High & $12(37.5)$ & $13(36.1)$ & .91 \\
\hline \multicolumn{4}{|l|}{ Admission, n (\%) } \\
\hline Acute (fracture) & $18(56.3)$ & $18(50.0)$ & .61 \\
\hline Elective (replacement) & $14(43.8)$ & $18(50.0)$ & .61 \\
\hline
\end{tabular}

* Range 0 (severe cognitive impairment) to 30 (no cognitive impairment).

${ }^{\dagger}$ Range 20/20 (no visual impairment) to 20/800 (severe visual impairment).

${ }^{\ddagger}$ Range 0 (no acute health problems) to 70 (severe acute health problems).

$\S$ Ratio greater than 18 indicates dehydration.

"Range 0 (depression not likely) to 15 (depression very likely).

"Range 0 (severe disability) to 20 (no disability).

$\mathrm{SD}=$ standard deviation.

These findings have important implications for the management of elderly patients at risk of delirium. Primary prevention (proactive geriatric consultation) is an effective strategy in preventing delirium, and even when delirium develops, there was still an additional effect of this pharmacological prophylactic intervention on the duration and severity of delirium. The results of this study are not in accord with the results of one other study, in which haloperidol prophylaxis led to a reduction of postoperative delirium in gastrointestinal patients and no data are available on the reduction of severity or delirium duration. ${ }^{18}$ The current study did not find an effect on the primary endpoint of postsurgery delirium. Rather, there was a significant effect on secondary end points (duration and severity of delirium). Dissimilarities between samples, design, and type of surgery may explain the apparent differences in study outcomes. One study consisted of a small $(\mathrm{N}=80)$ group of gastrointestinal surgery patients randomized, not blinded, for $5 \mathrm{mg}$ haloperidol per day or saline solution. ${ }^{18}$ Another found a significant improvement in the symptoms of delirium and cognition in patients with acquired immunodeficiency syndrome treated with low-dose haloperidol and chlorpromazine for delirium. ${ }^{37}$
Perhaps the findings of the current study indicate a "priming" effect (i.e., therapeutic blood serum levels of haloperidol were reached sooner once treatment of delirium was started).

The strengths of this study include the targeting of older people at (intermediate or high) risk of developing delirium for prophylactic treatment only. Patients at low risk were not randomized. Postoperative delirium occurred in only five $(4.1 \%)$ of them, which is much less than in the at-risk sample $(15.8 \%)$. The findings corroborate the prognostic model of one study, which is an important finding in and of itself and provides important validation of this risk system for this particular population. ${ }^{10}$ This enabled the restriction of the prophylactic pharmacological treatment to those patients who needed it, thus maximizing the efficiency and clinical relevance of the intervention. All patients were assessed daily using standardized and validated instruments. Outcome data were relatively complete, and few patients were lost to follow-up. Moreover, haloperidol prophylaxis was well tolerated, and the extensive clinical experience with this antipsychotic drug in combination with its low costs enhances the extent to which this

Table 3. Results of Patients Who Developed Delirium, According to Study Group: Intention-to-Treat Group

\begin{tabular}{|c|c|c|c|c|}
\hline \multirow[b]{2}{*}{ Delirium Characteristic } & $\begin{array}{l}\text { Haloperidol } \\
\quad(n=32)\end{array}$ & $\begin{array}{l}\text { Placebo } \\
(\mathrm{n}=36)\end{array}$ & \multirow{2}{*}{$\begin{array}{c}\text { Mean Difference } \\
\text { (95\% Confidence Interval) }\end{array}$} & \multirow[b]{2}{*}{$P$-value } \\
\hline & \multicolumn{2}{|c|}{ Mean \pm Standard Deviation } & & \\
\hline Highest Delirium Rating Scale score & $14.4 \pm 3.4$ & $18.4 \pm 4.3$ & $4.0(2.0-5.8)$ & $<.001$ \\
\hline Delirium duration, days & $5.4 \pm 4.9$ & $11.8 \pm 7.5$ & $6.4(4.0-8.0)$ & $<.001$ \\
\hline Hospital days & $17.1 \pm 11.1$ & $22.6 \pm 16.7$ & $5.5(1.4-2.3)$ & $<.001$ \\
\hline
\end{tabular}

Note: Because of rounding, percentages may not total 100. 


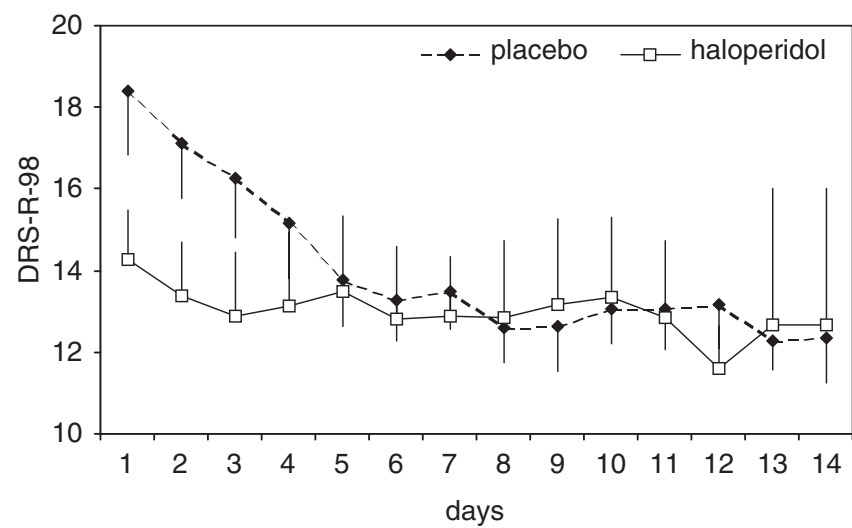

Figure 2. Mean of Delirium Rating Scale, revised version-98 (DRS-R-98) scores during delirium for treatment groups: $x$ axis $=$ days; $y$-axis $=$ mean DRS-R-98 scores of patients with delirium. The mean of the scores on the DRS-R-98 and the $95 \%$ confidence intervals in patients with delirium from the haloperidol $(n=32)$ and placebo $(n=36)$ groups, respectively. During each of the first 3 days after onset of delirium, the severity, as measured using the DRS-R-98, was significantly lower in patients who had received haloperidol perioperatively.

prevention protocol can be applied in other settings. The significantly lower severity and shorter duration of delirium, as well as the fewer total number of days haloperidol patients were hospitalized, than those receiving placebo strongly suggests that haloperidol prophylaxis contributed to the effectiveness of the intervention strategy. These findings support that, when applied to clinical practice, this strategy combining nonpharmacological and pharmacological interventions may lead to smaller numbers of patients who will have delirium and that it may shorten the severity and duration of this neuropsychiatric syndrome.

Some limitations of this study need to be addressed. This study was underpowered, given the relatively low delirium rates. The overall incidence of postoperative delirium was much lower than was expected based on the literature and previous experience in the study hospital. The



Figure 3. Proportion of patients suffering from delirium during the first 2 weeks postoperatively: $x$-axis $=$ days; $y$-axis $=$ proportion of patients with delirium. The flags indicate the upper and lower limits of the $95 \%$ confidence interval of this proportion in the haloperidol $(\mathrm{n}=212)$ and placebo $(\mathrm{n}=218)$ groups, respectively. During the entire postoperative period, this proportion was lower in patients who had received haloperidol prophylaxis; this difference was significant from Day 5 until Day 8 postoperatively. implementation of proactive geriatric consultation, provided to all the patients in both groups, may have stimulated the attention for predisposing and precipitating factors for delirium of the nursing and medical staff of the participating wards. In turn, it could have led to extra care for at-risk patients, thereby decreasing the likelihood of incident delirium. Such an effect may have caused the results to tend toward the null hypothesis. Second, the study included far more patients who were at intermediate risk than at high risk for delirium. This may have resulted in fewer incident cases of delirium, decreasing discriminating power. Third, patients were treated with a low dosage of haloperidol prophylaxis, which may explain the absence of a clear difference in the incidence of delirium between patients receiving haloperidol and placebo prophylaxis. The choice of $1.5 \mathrm{mg} / 24$ hours dosage of haloperidol was based on the average starting dose for treatment of delirious older people and the minimal chance of extrapyramidal side effects with a total daily dose of less than $3 \mathrm{mg} .{ }^{17,24}$ Another study found "extremely low" prevalence of extrapyramidal side effects with haloperidol and chlorpromazine treatment of delirium in patients with acquired immunodeficiency syndrome. ${ }^{37}$ Higher doses of haloperidol may perhaps be necessary for primary prevention of delirium, but the frequency and severity of side effects may increase, especially in vulnerable patient groups. A dose titration and duration study might have benefited this study. Fourth, the effect of the haloperidol prophylaxis could be solely due to reduction of certain symptoms of delirium (agitation or hyperactive symptoms), implying that haloperidol does not change the underlying nature or course of delirium. However, delirium was diagnosed based on clinical judgment and CAM ratings. A shorter duration of delirium implies a treatment effect beyond the psychomotor symptoms. The total score on the DRS-R-98 before the onset of delirium was not different between the haloperidol and placebo patients. The effect of sedation on psychomotor symptoms from haloperidol is therefore highly unlikely. Fifth, there was no information about prehospitalization mental status, such as from proxy report, because most of the informant questionnaires were not returned, so in the acute (fracture) patients, the study intake MMSE might not have been reflective. Nevertheless, the MMSE results did not differ in the fracture population from those of the elective patients. Sixth, the study group consisted of elderly orthopedic patients only, which may limit generalizability to other settings. Seventh, factors such as medical complications, pain, and use of medications that have central nervous system (CNS) side effects may have influenced the secondary outcome measures that proved to be significant-severity of delirium, duration of delirium, and number of days in the hospital. However, but this was a randomized, controlled study, and if there were confounding variables, they would have been expected to be present in both treatment conditions alike. Moreover, a retrospective review of the medical charts did not show any evidence of more complications, more pain, or the use of more painkillers or drugs with CNS side effects in any of the two treatment groups.

Despite all these effects, which may have tended to bias results toward the null hypothesis, the significant results support the effectiveness of the intervention. 
A formal cost-effectiveness analysis was beyond the scope of this study, but it is likely that the intervention proposed here has the potential to yield net savings in addition to reducing the burden for patients and caregivers. This clinical trial holds substantial promise for the prevention and reduction of delirium in hospitalized, nonsurgical, older patients too. Further studies are needed to determine whether similar beneficial effects can be observed in other settings. Moreover, the potential effect of haloperidol prophylaxis on other patient outcome measures, such as morbidity, mortality, institutionalization, and long-term cognitive functioning, deserves further study.

\section{ACKNOWLEDGMENTS}

The principle investigators designed this study, which the Medical Center Alkmaar funded. Kees J. Kalisvaart had full access to all the data in the study and takes responsibility for the integrity of the data and the accuracy of data analysis.

We would like to thank Piet Bartels, PhD (conception, design, data analysis), Adrie Steenhoek, $\mathrm{PhD}$ (conception and design), Tjeerd van der Ploeg, $\mathrm{PhD}$ (data analysis), Liesbeth Geiteman, RN (data acquisition), and Folkert K. de Boer (data acquisition) for their work on the study and the staff of the orthopedic and surgical units and emergency department for their constant alertness, help, and enthusiasm during the study.

Financial Disclosure: None.

Author Contributions: Kees J. Kalisvaart, MD: content; concept and design; acquisition of data; analysis and interpretation of data; drafting of the manuscript; critical revision of the manuscript for intellectual content; administrative, technical, and material support; supervision; statistical analysis. Jos F. M. de Jonghe, PhD: content, conception and design, analysis and interpretation of data, drafting of the manuscript, critical revision of the manuscript for intellectual content, supervision, statistical analysis. Marja J. Bogaards, PharmD: content; concept and design; acquisition of data; analysis and interpretation of data; critical revision of the manuscript for intellectual content; administrative, technical, material support; supervision. Ralph Vreeswijk, RN, MSc: content; acquisition of data; analysis and interpretation of data; drafting of the manuscript; critical revision of the manuscript for intellectual content; administrative, technical, material support. Toine C. G. Egberts, PhD: content, concept and design, analysis and interpretation of data, drafting of the manuscript, critical revision of the manuscript for intellectual content, supervision, statistical analysis. Bart J. Burger, $\mathrm{MD}, \mathrm{PhD}$ : content, concept and design, acquisition of data, drafting of the manuscript, critical revision of the manuscript for intellectual content, supervision. Piet Eikelenboom, MD, PhD: content, concept and design, acquisition of data, analysis and interpretation of data, critical revision of the manuscript for intellectual content, supervision. Willem A. van Gool, MD, PhD: content, analysis and interpretation of data, drafting of the manuscript, critical revision of the manuscript for intellectual content, supervision, statistical analysis, drafting of the figures.

Sponsor's Role: none.

\section{REFERENCES}

1. Dyer CB, Ashton CM, Teasdale TA. Postoperative delirium. A review of 80 primary data-collection studies. Arch Intern Med 1995;155:461-465.

2. Galanakis P, Bickel H, Gradinger R et al. Acute confusional state in the elderly following hip surgery: Incidence, risk factors and complications. Int J Geriatr Psychiatry 2001;16:349-355.

3. Lindesay J, Rockwood K, Rolfson DB. The epidemiology of delirium. In: Lindesay J, Rockwood K, Macdonald AJ, eds. Delirium in Old Age, 1st Ed. New York: Oxford University Press, 2002, pp 27-50.

4. Marcantonio ER, Flacker JM, Michaels M et al. Delirium is independently associated with poor functional recovery after hip fracture. J Am Geriatr Soc 2000;48:618-624.

5. Marcantonio ER, Simon SE, Bergmann MA et al. Delirium symptoms in postacute care: Prevalent, persistent, and associated with poor functional recovery. J Am Geriatr Soc 2003;51:4-9.

6. McCusker J, Cole MG, Dendukuri N et al. Does delirium increase hospital stay? J Am Geriatr Soc 2003;51:1539-1546.

7. O'Keeffe S, Lavan J. The prognostic significance of delirium in older hospital patients. J Am Geriatr Soc 1997;45:174-178.

8. Berggren D, Gustafson Y, Erikson B. Postoperative confusion after anaesthesia in elderly patients with femoral neck fractures. Anesth Analg 1987;66:497-504.

9. Williams-Russo P, Urquhart BL, Sharrock NE et al. Post-operative delirium: Predictors and prognosis in elderly orthopedic patients. J Am Geriatr Soc 1992; 40:759-767.

10. Inouye SK, Viscoli CM, Horwitz RI et al. A predictive model for delirium in hospitalized elderly medical patients based on admission characteristics. Ann Intern Med 1993;119:474-481.

11. Cole MG, Primeau FJ, Elie LM. Delirium: Prevention, treatment, and outcome studies. J Geriatr Psychiatry Neurol 1998;11:126-137.

12. Inouye SK, Bogardus ST Jr, Charpentier PA et al. A multicomponent intervention to prevent delirium in hospitalized older patients. N Engl J Med 1999;340:669-676.

13. Marcantonio ER, Flacker JM, Wright RJ et al. Reducing delirium after hip fracture: A randomized trial. J Am Geriatr Soc 2001;49:516-522.

14. Milisen K, Foreman MD, Abraham IL et al. A nurse-led interdisciplinary intervention program for delirium in elderly hip-fracture patients. J Am Geriatr Soc 2001;49:523-532.

15. O'Keeffe S. The prevention of delirium. In: Lindesay J, Rockwood K, Macdonald AJ, eds. Delirium in Old Age, 1st Ed. New York: Oxford University Press, 2002, pp 153-185.

16. Cole MG, Primeau F, McCusker J. Effectiveness of interventions to prevent delirium in hospitalized patients: A systematic review. Can Med Assoc J 1996;155:1263-1268.

17. Practice guideline for the treatment of patients with delirium. American Psychiatric Association. Am J Psychiatry 1999;156:1-20.

18. Kaneko T, Cai J, Ishikura T et al. Prophylactic consecutive administration of haloperidol can reduce the occurrence of postoperative delirium in gastrointestinal surgery. Yonaga Acta Med 1999;42:179-184.

19. Ikarashi Y, Takahashi A, Ishimaru H et al. Suppression of cholinergic activity via the dopamine D2 receptor in the rat striatum. Neurochem Int 1997;30:191-197.

20. Ikarashi Y, Takahashi A, Ishimaru H et al. Regulation of dopamine D1 and D2 receptors on striatal acetylcholine release in rats. Brain Res Bull 1997;43:107-115.

21. Itil T, Fink M. Anticholinergic drug-induced delirium: Experimental modification, quantitative EEG and behavioral correlations. J Nerv Ment Dis 2004; 143:492-507.

22. Platt MM, Breitbart W, Smith M et al. Efficacy of neuroleptics for hypoactive delirium. J Neuropsych Clin Noeurosc 2004;6:66-67.

23. Cummings JL. Physostigmine ameliorates the delusions of Alzheimer's disease. Biol Psychiatry 1993;33:536-541.

24. Hassan E, Fontaine DK, Nearman HS. Therapeutic considerations in the management of agitated or delirious critically ill patients. Pharmacotherapy 1998;18:113-129.

25. Inouye SK, Charpentier PA. Precipitating factors for delirium in hospitalized elderly persons. Predictive model and interrelationship with baseline vulnerability. JAMA 1996;275:852-857.

26. Knaus WA, Draper EA, Wagner DP et al. APACHE II. A severity of disease classification system. Crit Care Med 1985;13:818-829.

27. Folstein MF, Folstein SE, McHugh PR. 'Mini-mental state'. A practical method for grading the cognitive state of patients for the clinician. J Psychiatr Res 1975;12:189-198.

28. Jorm AF, Jacomb PA. The Informant Questionnaire on Cognitive Decline in the Elderly (IQCODE): Socio-demographic correlates, reliability, validity and some norms. Psychol Med 1989;19:1015-1022.

29. Hetherington R. The Snellen chart as a test of visual acuity. Psychol Forsch 1954:24:349-357. 
30. Yesavage JA, Brink TL, Rose TL et al. Development and validation of a geriatric depression screening scale: A preliminary report. J Psychiatr Res 1982;17:37-49.

31. Mahoney FI, Barthel DW. Functional evaluation: The Barthel index. Md State Med J 1965;14:61-65.

32. Barnes TR. A rating scale for drug-induced akathisia. Br J Psychiatry 1989; 154:672-676

33. Diagnostic and Statistical Manual of Mental Disorders, 4th Ed., Text Revision. Washington, DC: American Psychiatric Association, 2000.
34. Inouye SK, van Dyck CH, Alessi CA et al. Clarifying confusion: The confusion assessment method. A new method for detection of delirium. Ann Intern Med 1990;113:941-948.

35. Trzepacz PT, Baker RW, Greenhouse J. A symptom rating scale for delirium. Psychiatry Res 1988;23:89-97.

36. Cummings JL. Clinical Neuropsychiatry. Orlando, FL: Grune \& Stratton, 1985.

37. Breitbart W, Marotta R, Platt MM et al. A double-blind trial of haloperidol, chlorpromazine, and lorazepam in the treatment of delirium in hospitalized AIDS patients. Am J Psychiatry 1996;153:231-237. 